\title{
SITE SPECIFIC IDENTIFICATION OF N-LINKED GLYCOSYLATION IN PROTEINS BY LIQUID CHROMATOGRAPHY-ELECTROSPRAY IONIZATION TANDEM MASS SPECTROMETRY
}

\author{
IRINA PERDIVARA ${ }^{1,2}$, ROXANA ELENA IACOB ${ }^{2,3}$, \\ MICHAEL PRZYBYLSKI ${ }^{1}$, AND KENNETH B. TOMER ${ }^{2}$ \\ ${ }^{1}$ Laboratory of Analytical Chemistry, University of Konstanz, Germany \\ ${ }^{2}$ Laboratory of Structural Biology, Mass Spectrometry group, NIEHS/NIH, \\ Research Triangle Park, NC, USA \\ ${ }^{3}$ The Barnett Institute, Northeastern University, Boston, MA, USA
}

\begin{abstract}
Recently, we reported the characterization of the glycans attached at the 11 $\mathrm{N}$-glycosylation sites of Hepatitis C virus E2 envelope glycoprotein by tandem mass spectrometry. Infections caused by Hepatitis $\mathrm{C}$ virus represent the main cause of liver diseases such as hepatitis, cirrhosis and hepatocellular carcinoma. The N-linked sugars consist primarily of high mannose glycans, with structures ranging from the minimal core structure, $\mathrm{Man}_{3} \mathrm{GlcNAc}_{2}$ (Man3) up to 12 hexose residues attached to the GlcNAc-B(1-4)-GlcNAc core (depicted as $\mathrm{Hex}_{3} \mathrm{Man}_{9} \mathrm{GlcNAc}_{2}$ ). Furthermore, the site N41 (N423) was observed to contain complex type glycans with the structures Man3-GlcNAc and Man3-GlcNAcFuc, in addition to the high mannose population Man3 through Man6, while the site N48 (N430) was occupied exclusively with complex type glycans (Man3-Fuc, Man3-GlcNAcFuc and Man3-GlcNAc ${ }_{2}$ Fuc). The present contribution summarizes our experimental observations upon the factors which may have an impact on the CID tandem mass spectra of glycopeptides.
\end{abstract}

\section{Introduction}

Protein glycosylation represents an important post-translational event involved in cellular localization of proteins, folding, proteolytic stability and biological lifetime, signal transduction and protein-protein interactions [1-4]. Most of cellsurface and secreted proteins are modified by N-linked glycosylation [5] which occurs at the consensus sequence Asn-Xxx-Ser/Thr/Cys, where Xxx can be any amino acid except for proline [6,7]. Unlike other post-translational modifications (e.g., phosphorylation), N-glycans are expressed as a set of structural variations on a conserved pentasaccharide core $\left(\mathrm{Man}_{3} \mathrm{GlcNAc}_{2}\right)$. Thus, a specific $\mathrm{N}$-consensus site may be occupied by several different glycans (site microheterogeneity), whereas macroheterogeneity arises from various such N-motifs contained in a polyprotein sequence. Structural characterization of individual glycoforms and their relative site occupancies in glycoproteins has become increasingly important for understanding biological functions exhibited by this class of biomolecules, as well as for quality control of recombinant protein pharmaceuticals [8]. 
Recently, mass spectrometry (MS) using soft ionization methods $[9,10]$ has become an invaluable tool for the analysis of post-translational modifications, in particular for protein glycosylation [11-13]. Several approaches have become commonly used to address this issue: (i) analysis of the N-linked carbohydrates released from the protein by enzymatic procedures followed by derivatization of the glycan pool [14], or (ii) analysis of the glycopeptides formed by proteolytic degradation of the protein $[15,16]$. While the former provides a global picture of the glycans decorating the protein, the second strategy discloses information about the carbohydrate attachment site, which can be obtained using various tandem mass spectrometric methods (reviewed in [17]).

Recently, we reported the mass spectrometric characterization of the N-glycans attached on the Hepatitis C virus (HCV) E2 glycoprotein [18] (DOI: 10.1016/ j.jasms.2007.11.022). Hepatitis $\mathrm{C}$ viral infections represent the main cause of liver diseases in humans, including chronic hepatitis, cirrhosis or hepatocellular carcinoma. $\mathrm{HCV}$ is a small, enveloped positive-strand RNA virus belonging to the Flaviviridae family [19]. The polyprotein encoded in the HCV genome is co- and post-translationally processed by host and viral peptidases into four structural (Core, E1, E2 and p7) and five non-structural proteins [20]. The envelope proteins E1 and E2, containing 6 and 11 Asn-consensus motifs, respectively, are heavily glycosylated [21]. Using a combination of proteases (trypsin and $\alpha$-chymotrypsin) and nano-scale liquid chromatography in combination with collision induced dissociation (CID) tandem MS, it was possible to characterize the glycoforms associated with each of the 11 glycosylation sites. The major glycans at 9 of the 11 sites are exclusively of high mannose type, comprising species ranging from the minimal core structure $\mathrm{Man}_{3} \mathrm{GlcNAc}_{2}$ (Man3) up to 12 hexose residues elongating the chitobiose core $\left(\mathrm{Hex}_{3} \mathrm{Man}_{9} \mathrm{GlcNAc}_{2}\right)$, whereas two sites, N41 (N423) and N48 (N430) proved to be occupied with complex type oligosaccharides of the following sugar composition: Man3-GlcNAc and Man3-GlcNAcFuc at the site N41 and Man3-Fuc, Man3-GlcNAcFuc, Man3-GlcNAc ${ }_{2}$ Fuc at the site N48 [18]. Digestion by $\alpha$-chymotrypsin led to formation of glycopeptides of different amino acid compositions containing the same glycosylation site, whose identity was confirmed by tandem MS. However, at specific collision energies, the MS/MS analyses of these glycopeptides revealed that specific fragmentation pathways may be preferred depending on: (i) amino acid composition; length of the peptide; and (ii) nature of the glycan. The present contribution summarizes our experimental observations upon the factors which may have an impact on the CID tandem mass spectra of glycopeptides. 


\section{Experimental}

\subsection{MATERIALS}

HCV E2 envelope glycoprotein (recombinant) was purchased from Austral Bio-logicals (San Ramon, CA). Urea, dithiothreitol, iodoacetamide, 96\% formic acid, ammonium bicarbonate and ethanol were purchased from Sigma-Aldrich (St. Louis, MO). Sequencing grade-modified trypsin was obtained from Promega (Madison, WI). Chymotrypsin was purchased from Roche Diagnostics Corporation (Indianapolis, IN). Acetonitrile was purchased from Caledon Laboratories, Ltd. (Georgetown, Ontario). Purified water (17.8 M 2 ) was obtained from an in-house Hydro Picopure 2 system. All chemicals were used without further purification unless otherwise specified.

\subsection{SAMPLE PREPARATION}

HCV E2 protein expressed in Chinese hamster ovary (CHO) cells was reduced, alkylated and digested with either trypsin or chymotrypsin following the procedure described in [18].

\subsection{MASS SPECTROMETRY}

nanoLC/MS/MS analyses were performed on a Waters Q-Tof Premier mass spectrometer equipped with a nanoAcquity UPLC system and a NanoLockspray source (Waters, Milford, MA). Separations were performed using a $3 \mu \mathrm{m}$ nanoAquity Atlantis column dC18 $100 \mu \mathrm{m} \times 100 \mathrm{~mm}$ (Waters) at a flow rate of $300 \mathrm{nl} / \mathrm{min}$. A nanoAquity trapping column $5 \mu \mathrm{m} \mathrm{C} 18180 \mu \mathrm{m}$ x $200 \mathrm{~mm}$ (Waters) was positioned in-line with the analytical column. Trapping of a $2 \mu$ aliquot of the digested sample was performed for $3 \mathrm{~min}$ at $5 \mu \mathrm{l} / \mathrm{min}$ flow rate. Peptides were eluted using a linear gradient from $98 \% \mathrm{~A}$ (water/ $0.1 \%$ formic acid (v/v)) and $2 \% \mathrm{~B}$ (acetonitrile/ $0.1 \%$ formic acid $(\mathrm{v} / \mathrm{v}))$ to $95 \%$ B over 60 min. Mass spectrometer settings for MS analyses were as follows: capillary voltage of $3.5 \mathrm{kV}$, cone voltage of 30 $\mathrm{V}$, collision energy of $8.0 \mathrm{~V}$, and scan range of 200-2,000 Da. The tandem mass spectra were obtained in the data dependant acquisition mode, and a fixed range of collision energies between 30 and $40 \mathrm{~V}$ were applied in order to ensure good fragmentation of the glycopeptides. Peptides were identified by mass and charge measurement compared to the in silico digestion using the BioLynx Protein/ Peptide Editor, feature of MassLynx V4.0 (Micromass, UK). 


\section{Results and Discussion}

The amino acid sequence of the HCV E2 glycoprotein is shown in Figure 1, with the numbering starting from Ala1 to Lys333 (which corresponds to the numbering Ala383 to Lys715 within the entire HCV polyprotein of reference strain H, GenBank accession number 009606: this numbering is shown in parentheses). The 11 glycosylation sites, highlighted in bold, were found to be primarily occupied by high-mannose glycans at nine sites and by complex type glycans at the remaining two (N41 (N423) and N48 (N430)). The population of the observed high-mannose glycans was highly heterogeneous, containing structures ranging from $\mathrm{Man}_{3} \mathrm{GlcNAc}_{2}$ (Man3) up to $\mathrm{Hex}_{3} \mathrm{Man}_{9} \mathrm{GlcNAc}_{2}$ (Hex3Man9). The primary sequence of E2 contains only a few trypsin cleavage sites, and, therefore, longer proteolytic fragments, containing multiple glycosylation sites (which were not observed in MS), were formed when trypsin was used to generate the glycopeptides. Consequently, only 4 out of 11 sites were observed in the MS analysis of the tryptic digest. In order to elucidate the glycosylation pattern at the remaining sites, chymotrypsin was used to produce shorter proteolytic fragments, which would contain a single glycosylation position within each peptide.

\footnotetext{
1 AETHVTGGNA GRTTAGLVGL LTPGAKQNIQ LINTNGSWHI NSTALNCNES

51 LNTGWLAGLF YQHKFNSSGC PERLASCRRL TDFAQGWGPI SYANGSGLDE

101 RPYCWHYPPR PCGIVPAKSV CGPVYCFTPS PVVVGTTDRS GAPTYSWGAN

151 DTDVFVLNNT RPPLGNWFGC TWMNSTGFTK VCGAPPCVIG GVGNNTLLCP

201 TDCFRKYPEA TYSRCGSGPR ITPRCMVDYP YRLWHYPCTI NYTIFKVRMY

251 VGGVEHRLEA ACNWTRGERC DLEDRDRSEL SPLLLSTTQW QVLPCSFTTL

301 PALSTGLIHL HQNIVDVQYL YGVGSSIASW AIK
}

Figure 1. Amino acid sequence of the envelope glycoprotein E2, starting from amino acid 1 to 333 (383-715) from strain HCV-1a (GenBank accession number AF 009606). The 11 glycosylation sites are shown in bold

\subsection{CID OF GLYCOPEPTIDES WITH IDENTICAL PEPTIDE BACKBONE CONTAINING DISTINCT GLYCANS}

The site N41 (N423), observed in the chymotryptic peptide 39-45 (HIN*STAL), was found to be occupied by both high mannose and complex type glycans. The high mannose sugars comprise structures ranging from Man3 to Man6, whereas the structures determined for the two complex type glycans were Man3-GlcNAc and Man3-GlcNAcFuc, respectively. 

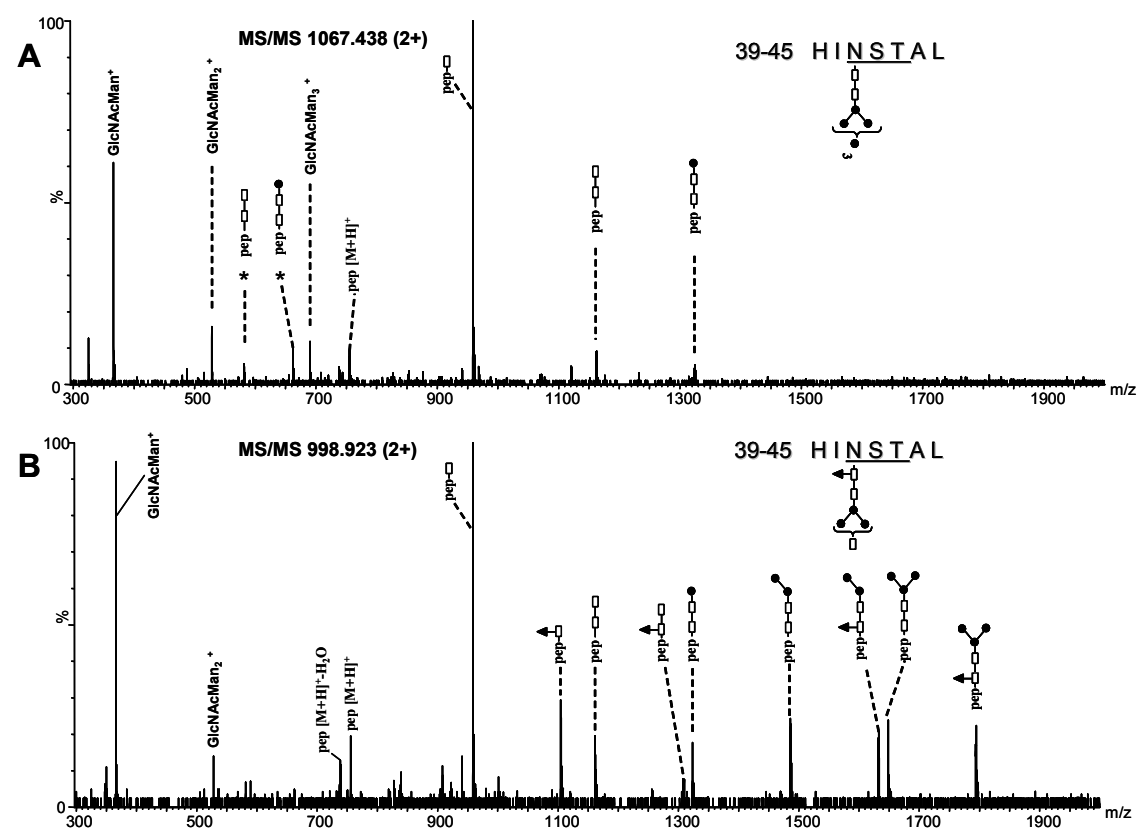

Figure 2. MS/MS spectra of the peptide 39-45 containing: A. a high mannose glycan of the structure Man6, and B. a complex type glycan of the structure Man3GlcNAcFuc. The spectra were obtained in the data dependent mode with a collision energy ramp from 30 to $40 \mathrm{~V}$. The ions indicated with an asterisk (*) are doubly charged, all the other ions are singly charged

Figure 2 shows the MS/MS analyzes of the peptide 39-45 containing the Man6 carbohydrate (Figure 2A) and the complex type glycan Man3-GlcNAcFuc (Figure 2B). The precursor ions of $m / z \quad 1,067.438$ and 998.923 respectively, are doubly charged while the majority of the fragment ions observed in these spectra are singly charged. The ion of $m / z 958.480$, representing the base peak in both tandem mass spectra, was assigned to the intact peptide 39-45 retaining the first $N$-acetyl glucosamine of the chitobiose core and allowed for the identification of the peptide carrying the glycan 39-45 with a the mass accuracy of $5 \mathrm{ppm}$. Complete loss of the glycan moiety was observed, as the singly charged ion $\mathrm{m} / \mathrm{z} 755.398$ represents the singly protonated aglycone.

The ions observed in the spectra shown in Figure 2 result from B/Y-type fragmentations of the oligosaccharide (nomenclature after Domon and Costello [22]) and are accompanied by charge reduction of the precursor ion. An additional fragmentation pathway, arising primarily from the loss of the core fucose with charge reduction, is observed in the MS/MS of the glycopeptide containing the complex glycan. 


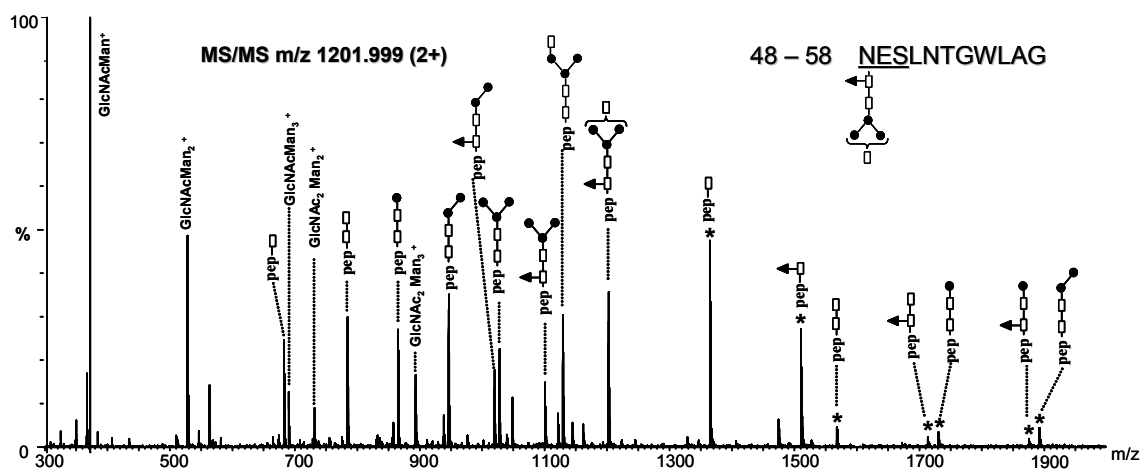

Figure 3. MS/MS spectrum of the doubly protonated precursor of $m / z \quad 1,201.95$, representing the peptide 48-58 containing the glycan Man3GlcNAcFuc. The spectrum was obtained in the data dependent mode by ramping the collision energy from 30 to $40 \mathrm{~V}$. The singly charged ions containing the peptide backbone are indicated with an asterisk $(*)$

The MS/MS spectrum of the doubly protonated glycopeptide $m / z 1,201.999$, which corresponds to the amino acid sequence 48-58 containing the glycosylation site N48 (N430) which is occupied by complex glycan of the same composition as that on peptide $39-45$, is shown in Figure 3. This glycopeptide was formed by non-specific chymotrypsin activity, which cleaved the protein backbone at the alkylated Cys47 and Gly58. As in the MS/MS spectrum of glycopeptide 39-45, singly charged fragments arise from B/Y-type fragmentations and allow for the identification of the glycan composition. Another series, however, of doubly charged ions is observed that arise by the sequential loss of neutral monosaccharides from the non-reducing end with the charge on the remaining Y-fragment. These results suggest that the decomposition of glycopeptides under identical fragmentation conditions can be dependent on: (i) the composition and identity of the glycan, because the presence of the labile fucose on the chitobiose core introduces a new fragmentation channel which competes with the fragmentation from the nonreducing end, and (ii) amino acid composition and length of the glycopeptide, as the longer peptides more readily retain multiple charges.

\subsection{CID OF GLYCOCONJUGATES WITH IDENTICAL GLYCANS ATTACHED TO THE SAME SITE IN DISTINCT PEPTIDES}

The glycosylation site N263 (N645), carrying exclusively high mannose glycans of composition Man5 to Man9, was observed in two glycopeptides, 246-272 and 251-264, respectively. Figure 4 shows the MS/MS spectra obtained for the triply charged precursor ions of $\mathrm{m} / z$ 1,547.855 and 992.743 of these glycopeptides, both having the carbohydrate Man6 attached at the asparagine residue. The dominating fragments in Figure 4A are the sugar oxonium ions resulting from processing of 
the glycan from the non-reducing end and the doubly charged glycopeptide ion containing a single $\mathrm{N}$-acetyl glucosamine (which is the base peak in this spectrum). The complete series of singly charged sugar fragments provides structural information about the oligosaccharide attached to the peptide.

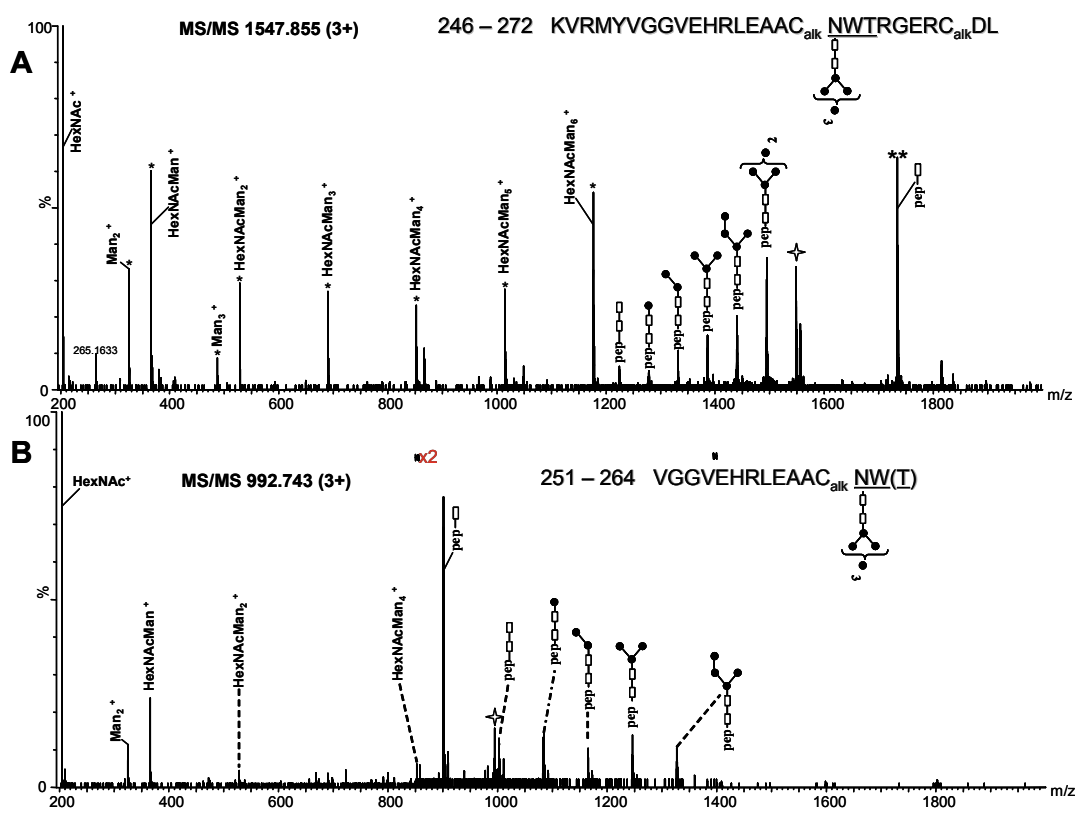

Figure 4. MS/MS spectra of glycopeptides containing the site N263 (N645) and a high mannose glycan of composition Man6: (A) triply charged precursor of $m / z$ 1,547.855 assigned to the glycopeptide 246-272. The precursor ion is highlighted with a star. Singly charged sugar oxonium ions are indicated with an asterisk $\left(^{*}\right)$. Doubly charged ions are depicted with a double asterisk $(* *)$. The ions in the range $m / z(1,200-1,600)$ are triply charged; (B) triply charged precursor of $\mathrm{m} / \mathrm{z} 992.840$ assigned to the glycopeptide 251-264. The precursor is indicated with a star. The ions in the range $m / z(900-1,400)$ (two times magnified) are doubly charged. The spectra were obtained with a collision energy ramp from 30 to $40 \mathrm{~V}$

The triply charged fragment ions in the range $m / z(1,200-1,600)$ arise from stepwise neutral loss of the individual monosaccharides from the free oligosaccharide end, with the minimal glycan retained on the peptide backbone being chitobiose. Except for the most abundant doubly protonated molecular species in this spectrum, observed at $\mathrm{m} / \mathrm{z} 1,733.430$, no significant fragmentation with charge reduction of the precursor was observed. By comparison, the MS/MS shown in Figure $4 \mathrm{~B}$ contains almost exclusively doubly charged fragments resulted from the charge reduction fragmentation pathway. Compared with Figure 4A, only a few sugar oxonium ions of low abundance were observed. In the MS/MS spectrum of 
glycopeptide 246-272, the fragment ion containing the first $\mathrm{N}$-acetyl glucosamine residue, formed by $\mathrm{B} / \mathrm{Y}$-type fragmentation and charge reduction, represents the most abundant ion in this spectrum.

Peptide 246-272, compared to peptide 251-264, extends five amino acids longer at the N-terminus (two of which are basic residues) and seven amino acids longer at the $\mathrm{C}$-terminus. This may explain the enhanced probability for retention of three charges on the fragment ions observed in Figure 4A in the mass range $1,200-1,600$. Thus, it appears that the amino acid composition and the length of the peptide containing the glycan determine the dominant decomposition pathways of glycoconjugates.

\subsection{PEPTIDE BACKBONE FRAGMENTATION (IN THE CID OF GLYCOCONJUGATES)}

A major characteristic of the CID spectra of glycopeptides is that fragment ions resulting from processing of the glycan [17] are primarily observed, whereas the formation of backbone fragments is less common. While fragmentation of the glycan moiety is achieved using low collision energies (typically 15-25 V), decomposition of the peptide requires elevated collision energies. Wuhrer et al. reported the observation of abundant backbone fragmentation using collision energies of 30 $\mathrm{V}$ in a quadrupole-FTICR instrument. Under these conditions, however, fragmentation of the glycan was complete and fragment ions due to successive loss of glycans were no longer observed [17]. Other groups used collision energies up to 80 $\mathrm{V}$ in order to observe fragmentation of the peptide [15].

The glycosylation site N174 (N556), observed in the chymotryptic peptide $173-178$ and in the peptide $171-180$, formed by non-specific activity of the enzyme, is occupied with a heterogeneous population of high mannose glycans, ranging from the minimal core structure Man3 up to nine mannoses attached to the chitobiose core (Man9). Representative MS/MS spectra of the glycopeptides 173-178 and 171-180, containing the Man6 oligosaccharide are shown in Figure 5. These spectra were obtained with a collision energy ramp from 30 to $40 \mathrm{~V}$ and in both the most abundant ions were assigned the structure of the peptide retaining the first GlcNAc rest. The MS/MS spectrum in Figure 5A displays an abundant ion corresponding to the protonated peptide 173-178, which was formed by the complete loss of the oligosaccharide chain. Remarkably, the MS/MS spectrum of the shorter peptide 173-178 (Figure 5A) contains abundant $\mathrm{b}$ and y peptide fragments, while no such fragments were observed in the MS/MS spectrum of the peptide 171-180. The latter peptide contains four additional amino acids compared to peptide 173-178 (Figure 5B). The MS/MS spectrum of the precursor ion of $m / z$ $1,275.970$ (Figure 5B) consists primarily of ions derived from fragmentation of the glycan retained on the intact peptide. The majority of the observed of the b-type backbone fragment ions $\left(b_{3}-m / z 333.100, b_{4}-m / z 434.151, b_{5}-m / z\right.$ 491.172), which contain the $\mathrm{N}$-glycosylation site, were also found accompanied by ions retaining 

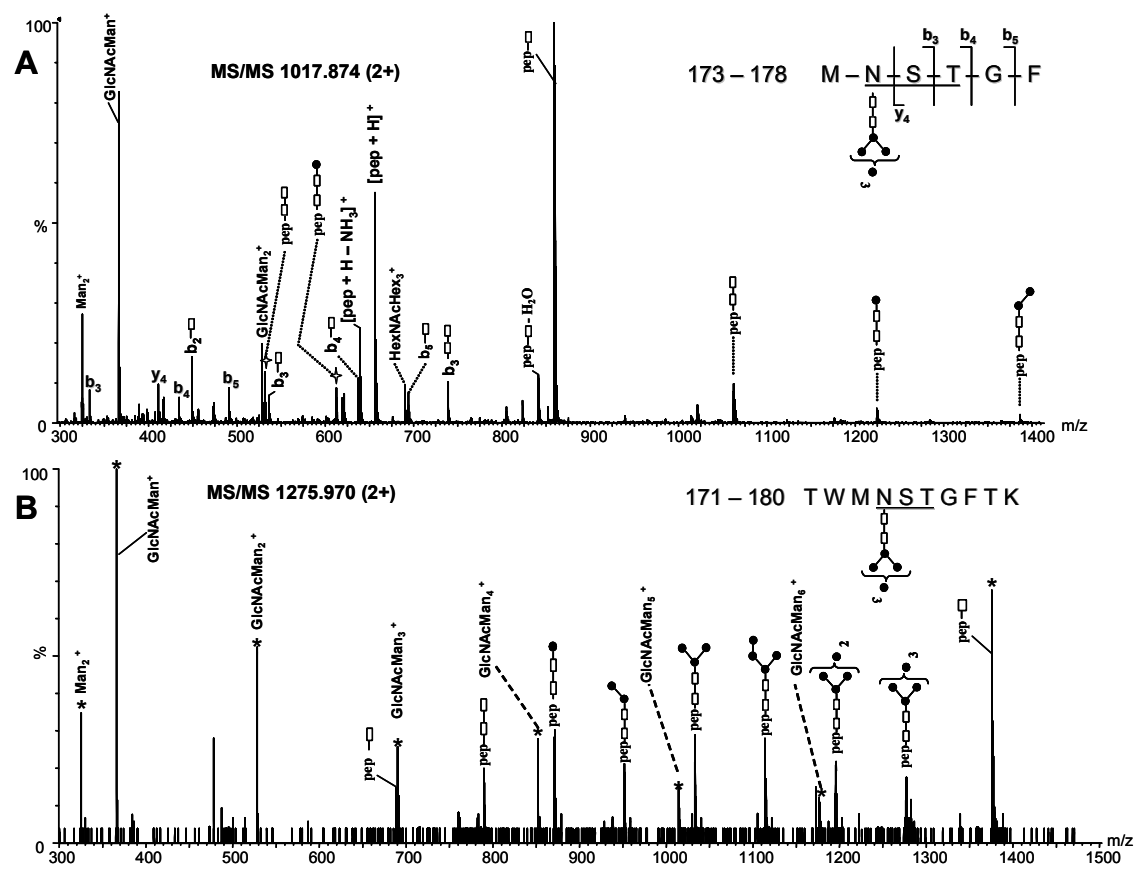

Figure 5. MS/MS spectra of doubly protonated glycopeptide species containing the glycosylation site N174 (N556); (A) precursor ion $\mathrm{m} / z$ 1,017.83. The ions highlighted with a star are doubly charged; (B) precursor ion $m / z$ 1,276.48. The ions indicated with an asterisk (*) are singly charged. The spectra were obtained with a collision energy ramp from 30 to $40 \mathrm{~V}$

the first $\mathrm{N}$-acetyl glucosamine residue $\left(\mathrm{b}_{3}-\mathrm{GlcNAc} \mathrm{m} / \mathrm{z} 536.221, \mathrm{~b}_{4}-\mathrm{GlcNAc} \mathrm{m} / \mathrm{z}\right.$ $637.210, b_{5}-$ GlcNAc $m / z$ 694.262) and, to a lesser extent, the second GlcNAc $\left(b_{3}-\right.$ $\mathrm{GlcNAc}_{2}-m / z$ 739.242). These ions confirmed the sugar attachment site.

The observation of peptide fragments which partially retain $N$-acetyl glucosamine is in agreement with data published by other groups [15,17], which showed that the GlcNAc was retained on the y-ions. Their data suggests that the formation of such fragments, however, is dependent on the amino acid composition and location of the glycosylation site within the peptide.

Another example in which backbone fragmentation was observed is illustrated in the MS/MS spectrum of the glycopeptide 235-242 containing the site N241 (N623) (Figure 6). Although the length of this peptide is comparable with that of the peptide 173-178, only two peptide fragments were observed, $\mathrm{b}_{6}$ at $\mathrm{m} / \mathrm{z} 715.351$ and $\mathrm{y}_{6}$-GlcNAc at $\mathrm{m} / z$ 913.381, probably because the formation of the $\mathrm{y}_{6}$ ion is enhanced by the presence of the amino acid motif YP [23]. 


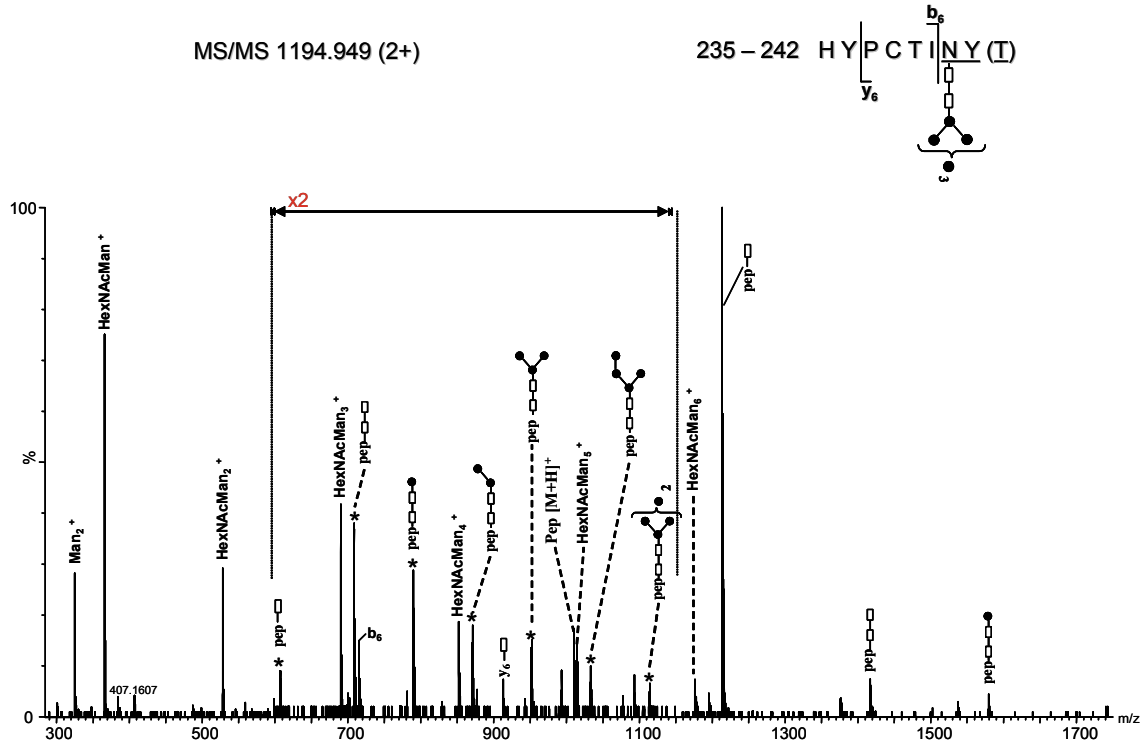

Figure 6. MS/MS spectrum of the doubly charged precursor ion of $\mathrm{m} / \mathrm{z} 1,194.898$, corresponding to the glycopeptide 235-242 containing the Man6 glycan. For clarity, the $\mathrm{m} / \mathrm{z}$ region $600-1,150$ was magnified by two. Doubly charged ions are indicated with an asterisk $\left(^{*}\right)$. The spectrum was obtained with a collision energy ramp from 30 to $40 \mathrm{~V}$

Thus, under identical collision energy conditions, the observation of backbone frag-mentation appears to be dependent on the amino acid composition of the glycopeptide.

\section{Conclusions}

Several examples of tandem mass spectra of N-glycopeptides derived from the HCV E2 envelope glycoprotein, which contain neutral oligosaccharides, were presented and all contain abundant ions resulted from the fragmentation of the glycan from the non-reducing end. Optimal decomposition of the glycopeptides was achieved using a collision energy ramp from 30 to $40 \mathrm{~V}$. The most abundant ion in all spectra corresponds, almost exclusively, to the intact peptide retaining one $\mathrm{N}$-acetyl glucosamine residue. Four major pathways for fragmentation of the glycopeptides were observed: (i) neutral loss of the individual monosaccharides from the free end of the sugar with charge retention on the remaining Y-ion; (ii) subsequent loss of the monosaccharide residues with charge reduction of the precursor ion and/or characteristic formation of sugar oxonium ions (B-ions); (iii) if the glycan contains a fucose, neutral loss of the fucose residue competes with neutral loss from the non-reducing end; (iv) cleavage of the peptide backbone. From our data 
and from the results published by other groups, the rate of each process was observed to depend on several factors, such as: composition of the glycan, number and amino acid sequence of the peptide and the amount of collision energy and conditions must be optimized for each individual experiment in order to obtain optimal information.

\section{References}

1. Marsh, M. and A. Helenius, Virus entry: open sesame. Cell, 2006. 124(4): 729-40.

2. Slawson, C., M.P. Housley, and G.W. Hart, O-GlcNAc cycling: how a single sugar posttranslational modification is changing the way we think about signaling networks. J Cell Biochem, 2006. 97(1): 71-83.

3. Smith, A.E. and A. Helenius, How viruses enter animal cells. Science, 2004. 304(5668): $237-42$.

4. Wormald, M.R. and R.A. Dwek, Glycoproteins: glycan presentation and protein-fold stability. Structure, 1999. 7(7): R155-60.

5. Apweiler, R., H. Hermjakob, and N. Sharon, On the frequency of protein glycosylation, as deduced from analysis of the SWISS-PROT database. Biochim Biophys Acta, 1999. 1473(1): 4-8.

6. Kornfeld, R. and S. Kornfeld, Assembly of asparagine-linked oligosaccharides. Annu Rev Biochem, 1985. 54: 631-64.

7. Gavel, Y. and G. von Heijne, Sequence differences between glycosylated and nonglycosylated Asn-X-Thr/Ser acceptor sites: implications for protein engineering. Protein Eng, 1990. 3(5): 433-42.

8. Srebalus Barnes, C.A. and A. Lim, Applications of mass spectrometry for the structural characterization of recombinant protein pharmaceuticals. Mass Spectrom Rev, 2007. 26(3): 370-88.

9. Fenn, J.B., et al., Electrospray ionization for mass spectrometry of large biomolecules. Science, 1989. 246(4926): 64-71.

10. Hillenkamp, F., et al., Matrix-assisted laser desorption/ionization mass spectrometry of biopolymers. Anal Chem, 1991. 63(24): 1193A-1203A.

11. Wuhrer, M., A.M. Deelder, and C.H. Hokke, Protein glycosylation analysis by liquid chromatography-mass spectrometry. J Chromatogr B Analyt Technol Biomed Life Sci, 2005. 825(2): 124-33.

12. Zaia, J., Mass spectrometry of oligosaccharides. Mass Spectrom Rev, 2004. 23(3): 161-227.

13. Huddleston, M.J., M.F. Bean, and S.A. Carr, Collisional fragmentation of glycopeptides by electrospray ionization LC/MS and LC/MS/MS: methods for selective detection of glycopeptides in protein digests. Anal Chem, 1993. 65(7): 877-84.

14. Ciucanu, I., Per-O-methylation reaction for structural analysis of carbohydrates by mass spectrometry. Anal Chim Acta, 2006. 576(2): 147-55.

15. Harazono, A., et al., Site-specific N-glycosylation analysis of human plasma ceruloplasmin using liquid chromatography with electrospray ionization tandem mass spectrometry. Anal Biochem, 2006. 348(2): 259-68.

16. Wuhrer, M., et al., IPSE/alpha-1, a major secretory glycoprotein antigen from schistosome eggs, expresses the Lewis X motif on core-difucosylated N-glycans. Febs J, 2006. 273(10): 2276-92.

17. Wuhrer, M., et al., Glycoproteomics based on tandem mass spectrometry of glycopeptides. J Chromatogr B Analyt Technol Biomed Life Sci, 2007. 849(1-2): 115-28. 
18. Iacob, R., Perdivara, I., Przybylski, M., and Tomer, K.B., Mass spectrometric characterization of glycosylation of Hepatitis C virus E2 envelope glycoprotein reveals extended microheterogeneity of N-glycans. JASMS, 2007.

19. Rehermann, B. and M. Nascimbeni, Immunology of hepatitis B virus and hepatitis C virus infection. Nat Rev Immunol, 2005. 5(3): 215-29.

20. Randall, G. and C.M. Rice, Hepatitis $C$ virus cell culture replication systems: their potential use for the development of antiviral therapies. Curr Opin Infect Dis, 2001. 14(6): 743-7.

21. Duvet, S., et al., Hepatitis $\mathrm{C}$ virus glycoprotein complex localization in the endoplasmic reticulum involves a determinant for retention and not retrieval. J Biol Chem, 1998. 273(48): 32088-95.

22. Domon, B. and Costello, C.E., A Systematic Nomenclature for Carbohydrate Fragmentations in FAB-MS/MS Spectra of Glycoconjugates. Glycoconjugate J, 1988. 5: 397-409.

23. Paizs, B. and S. Suhai, Fragmentation pathways of protonated peptides. Mass Spectrom Rev, 2005. 24(4): 508-48 